\title{
The problematic connection between low-luminosity gamma-ray bursts and ultra-high-energy cosmic rays
}

\author{
Filip Samuelsson, ${ }^{a, *}$ Damien Bégué,${ }^{b}$ Felix Ryde, ${ }^{a}$ Asaf Pe'er ${ }^{b}$ and Kohta \\ Murase $^{c, d, e, f}$ \\ ${ }^{a}$ Department of Physics, KTH Royal Institute of Technology, \\ and The Oskar Klein Centre, SE-10691 Stockholm, Sweden \\ ${ }^{b}$ Department of Physics, Bar-Ilan University, Ramat-Gan 54900, Israel \\ ${ }^{c}$ Department of Physics, The Pennsylvania State University, University Park, Pennsylvania 16802, USA \\ ${ }^{d}$ Department of Astronomy \& Astrophysics, The Pennsylvania State University, \\ University Park, Pennsylvania 16802, USA \\ ${ }^{e}$ Center for Particle and Gravitational Astrophysics, The Pennsylvania State University, \\ University Park, Pennsylvania 16802, USA \\ ${ }^{f}$ Yukawa Institute for Theoretical Physics, Kyoto, \\ Kyoto 606-8502 Japan \\ E-mail: filipsamakth.se
}

The origin of ultra-high-energy cosmic rays (UHECRs) remains debated. The prompt and afterglow phases of low-luminosity gamma-ray bursts (LLGRBs) are seen as promising candidates for this acceleration. Here, we investigate this connection by looking at the unavoidable emission from the electrons that are co-accelerated together with UHECRs. Specifically, we use the data from the archetypical low-luminosity GRB 060218. We find that if acceleration of UHECRs occurred during the prompt phase, the emission from the electrons would be orders of magnitude brighter than the observations in the optical band. For the afterglow phase, we limit the total available kinetic energy by comparing the emission from the thermal electrons to the radio data at three days. We find that the total energy in the afterglow is not sufficient to supply the UHECR flux observed at Earth. These results challenge the mildly relativistic outflows of LLGRBs as the main sources of UHECRs.

$37^{\text {th }}$ International Cosmic Ray Conference (ICRC 2021)

July 12 th - 23rd, 2021

Online - Berlin, Germany

\footnotetext{
${ }^{*}$ Presenter
} 


\section{Introduction}

The origin of ultra-high-energy cosmic rays (UHECRs) remains a mystery despite intensive research. Due to their electric charge, UHECRs are bent in the interstellar and intergalactic magnetic fields and, thus, do not point back to their source. To understand what astrophysical objects are responsible for their immense acceleration, other diagnostics have to be considered. Specifically, a lot of information can be obtained by looking at other messenger types.

Here, we consider the prompt and afterglow phases of low-luminosity GRBs (LLGRBs) as possible sources for UHECRs. Our aim is to characterize the inevitable electromagnetic emission from the electrons that are also accelerated in the UHECR acceleration region, and to compare this emission to observations. The emission from the co-accelerated electrons is a powerful tool that can constrain possible UHECR sources [1]. It also contains complementary information to other emitted messengers, such as neutrinos and photohadronic gamma-rays, and is therefore important to consider when conducting multi-messenger studies of UHECR sources. The methodology and results presented here are described in more detail in Samuelsson et al. [1, 2].

\section{GRB 060218 as a proxy}

We study LLGRBs using data for the archetypical low-luminosity GRB 060218 as a proxy for the population. GRB 060218 was studied extensively because of its proximity and association with SN 2006aj [3,4]. The main motivation to this event as a proxy for the population is the high quality of the available data for GRB 060218 and that this burst is often used as the canonical LLGRB in UHECR and neutrino studies [see e.g., 5]. Currently, only a handful of LLGRBs have been detected. In its prompt optical flux and afterglow radio flux, which are the two most important observables for our study, GRB 060218 seems to be similar to other LLGRBs where these data are available [6-8]. This indicates that the conclusions we find may be representable for the whole sample. However, we note that if GRB 060218 is found to be an outlier of the sample when more detections become available, the study should be remade with updated values for the fluxes.

\section{UHECR acceleration during the prompt phase}

For UHECR acceleration to be possible, the timescale for acceleration has to be shorter than the timescales for relevant cooling processes. Considering energy losses due to synchrotron emission, adiabatic cooling, and photohadronic interactions, one gets $t_{\mathrm{acc}}^{\prime}<\min \left[t_{\mathrm{sync}}^{\prime}, t_{\mathrm{ad}}^{\prime}, t_{p \gamma}^{\prime}\right]$, where the primes denote that the timescales are evaluated in the comoving frame. As the timescales are functions of the particle energy $E^{\prime}$, the comoving magnetic field strength $B^{\prime}$, and the radius from the progenitor $r$, one can calculate the maximum observed energy $E$ from the requirement $t_{\mathrm{acc}}^{\prime}<\min \left[t_{\mathrm{sync}}^{\prime}, t_{\mathrm{ad}}^{\prime}, t_{p \gamma}^{\prime}\right]$. The maximum observed energy as a function of $B^{\prime}$ and $r$ is shown in Figure 1 in the left-hand panel. From the panel, it is evident that there exists quite a large parameter space where acceleration to the highest observed energies of $10^{20} \mathrm{eV}$ is indeed possible. The figure is made considering a completely stripped iron nucleus, as this is the most easily accelerated particle and, thus, shows the least constraining scenario. Figure 1 is made for a bulk Lorentz factor of $\Gamma=10$. 

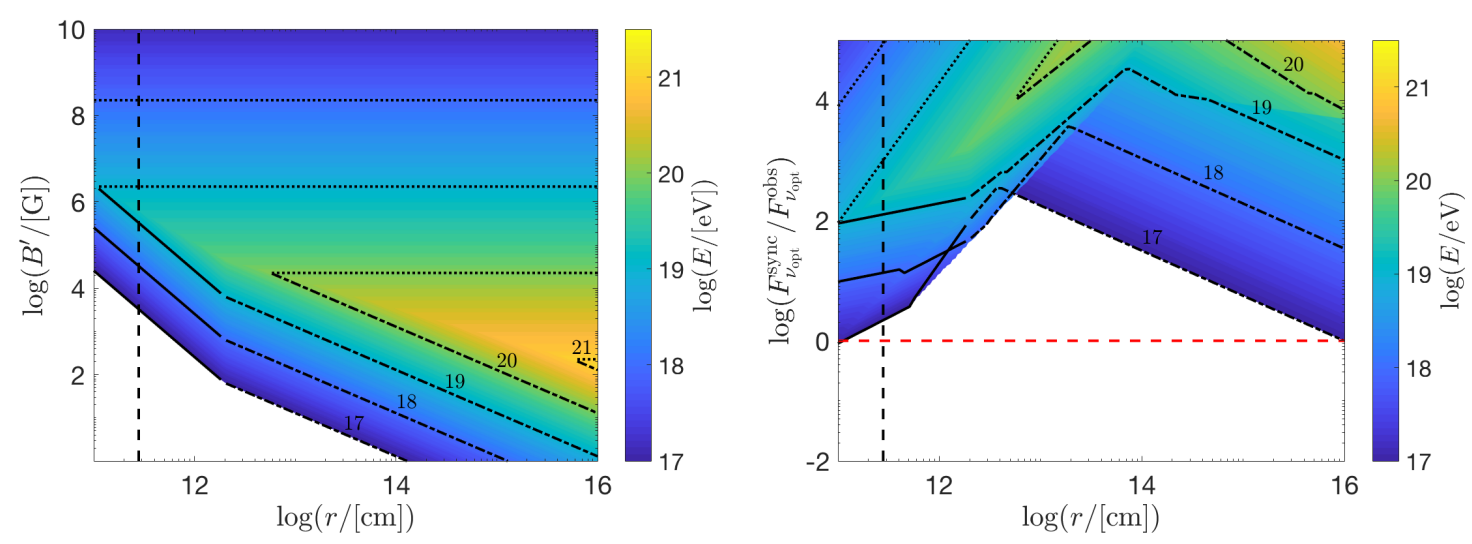

Figure 1: (Left) Maximum observed UHECR energy $E$ as a function of $B^{\prime}$ and $r$. The figure is for completely stripped iron and bulk Lorentz factor $\Gamma=10$. (Right) Predicted synchrotron flux from the co-accelerated electrons in the optical band as a function of $E$ and $r$. The flux has been normalized to the de-absorbed optical flux in GRB 060218. Everything above the red dashed line predicts brighter synchrotron emission than what was observed, and is therefore ruled out. The solid, dot-dashed, and dotted lines show the limits from the photohadronic interactions, the adiabatic expansion, and the synchrotron emission, respectively. The black dashed vertical line indicates the photosphere. More details can be found in Samuelsson et al. $[1,2]$.

Having determined the necessary magnetic field strength for UHECR acceleration, we continue by estimating the synchrotron flux from the electrons that are present at the acceleration region. The synchrotron spectrum is theoretically well determined [see e.g., 9] and the flux, which is a function of the magnetic field, can therefore be calculated. In the right-hand panel of Figure 1, we show the predicted optical flux from the electrons as a function of radius and the observed UHECR energy. The flux has been normalized to the de-absorbed optical flux for GRB 060218: $F_{v_{\mathrm{opt}}}^{\mathrm{obs}}=5.5 \times 10^{-27} \mathrm{erg} \mathrm{cm}^{-2} \mathrm{~s}^{-1} \mathrm{~Hz}^{-1}$ [10]. For the emission to be compatible with the data, the predicted flux has to be at the red dashed line or lower; everything above would overshoot the optical data. As can be seen in the panel, if GRB 060218 hosted accelerated of cosmic-rays to observed energies of $10^{20} \mathrm{eV}$, the optical synchrotron emission from the electrons co-accelerated with the UHECRs would be four orders of magnitude brighter than what was observed. We refer to Samuelsson et al. [2] for details on how the optical flux is calculated and how the results varies with the parameters.

\section{UHECR acceleration during the afterglow phase}

To constrain possible UHECR acceleration during the afterglow phase of GRB 060218, we calculate the necessary total energy of the blast wave. Given the observed flux of UHECRs at Earth, one can estimate the UHECR energy injection as $\sim 10^{44} \mathrm{erg} \mathrm{Mpc}^{-3} \mathrm{yr}^{-1}$ [11]. With an estimated local event rate of LLGRB of $10^{2}-10^{3} \mathrm{Gpc}^{-3} \mathrm{yr}^{-1}$ and with $10 \%$ of the total energy released as UHECRs, the total energy of the blast wave has to be $10^{51} \mathrm{erg}$ to supply the observed flux of UHECRs. Note that we this estimate is conservative, so $10^{51} \mathrm{erg}$ should be seen as the minimum 
energy required. This value of the total energy is higher than that found by previous studies of the afterglow of GRB 060218 [12-14]. However, there exists a degeneracy in the afterglow diagnostics. If the number fraction of electrons acceleration $\xi_{\mathrm{a}}$ is lower, the total kinetic energy can be higher and the signatures of the non-thermal emission would be the same [15]. Therefore, GRB 060218 might have had a sufficiently energetic afterglow.

The way to break the degeneracy is to look at the emission from the population of thermal electron $\left(1-\xi_{\mathrm{a}}\right)$, which will constitute the bulk number when $\xi_{\mathrm{a}}$ is small. To correctly capture the emission and absorption from the non-thermal and the thermal electrons, we use the numerical simulation outlined in Pe'er \& Waxman [16] with some necessary additions as explained in Samuelsson et al. [2]. The code assumes spherically symmetry and one-zone emission, accounting for cyclo-synchrotron emission, inverse Compton cooling of the electrons with full Klein-Nishina corrections, and pair production and annihilation. It smoothly interpolates between the relativistic Blandford-McKee solution and the nonrelativistic Sedov-Taylor solution.

Simulated spectra for the forward shock at three days with a blast wave energy of $10^{51} \mathrm{erg}$ for different values of $\xi_{\mathrm{a}}$ are shown by solid lines in Figure 2. The spectra are shown compared to data at $\sim 3$ days in radio, optical, and X-rays. The red X-ray and optical data are taken from Campana et al. [3], while the black and magenta radio data are taken from Kaneko et al. [17] and Soderberg et al. [13], respectively. Error bars are given as $1 \sigma$, except the upper limit (inverted magenta triangle) which is $3 \sigma$. The first hump at $\sim 10^{12} \mathrm{~Hz}$ is the synchrotron emission from the thermal electrons and the second hump around the optical band is the rising supernova. From the figure, one can see that, in the optical and X-ray band, the simulated spectra can become more or less consistent with the data by lowering $\xi_{\mathrm{a}}$. However, the emission in the radio band is always inconsistent with the radio data, regardless of how small $\xi_{\mathrm{a}}$ becomes. That is because the emission in the radio band is dominated by the thermal electrons, and the flux does not change much if number fraction of thermal electrons is 0.9 or 0.999 . The black dashed line is a simulated spectrum that has identical parameters to the black $\xi_{\mathrm{a}}=0.1$ spectrum, except that the blast wave energy has been lowered to $10^{49} \mathrm{erg}$. In this case, we do not overshine the radio data. Note that we are not trying to fit the spectrum to the data, we are only investigating whether a parameter set is compatible with the data or not.

The result shown in Figure 2 is dependent on the microphysics of the shock. For Figure 2, the fraction of internal energy given to electrons and magnetic fields were $\epsilon_{e}=10^{-1}$ and $\epsilon_{B}=10^{-3}$, respectively. To quantify how the result varies with $\epsilon_{e}$ and $\epsilon_{B}$ we calculate the ratio of the spectra generated by the code to the most constraining data point at $22.5 \mathrm{GHz}$ including its $1 \sigma$ error as $^{1}$

$$
R=\frac{F_{\text {radio }}^{\text {code }}}{F_{\text {radio }}^{\text {measud }}+F_{\text {radio }}^{\text {error }}} .
$$

In Figure 3, we show how $R$ varies with $\epsilon_{e}$, and $\epsilon_{B}$. The thick, dotted black line shows were the simulated spectra are compatible with the observations. The vertical dashed cyan line shows the value of $\epsilon_{e}$ found in PIC simulations of mildly relativistic shocks [18]. With this value of $\epsilon_{e}$, Figure 3 shows that the radio data, including its $1 \sigma$ error, would be overshone by almost two orders of magnitude regardless of the value of $\epsilon_{B}$.

\footnotetext{
${ }^{1}$ As evident from Figure 2, the value of $\xi_{\mathrm{a}}$ has little effect in the radio band. For Figure 3, we put $\xi_{\mathrm{a}}=0.01$.
} 


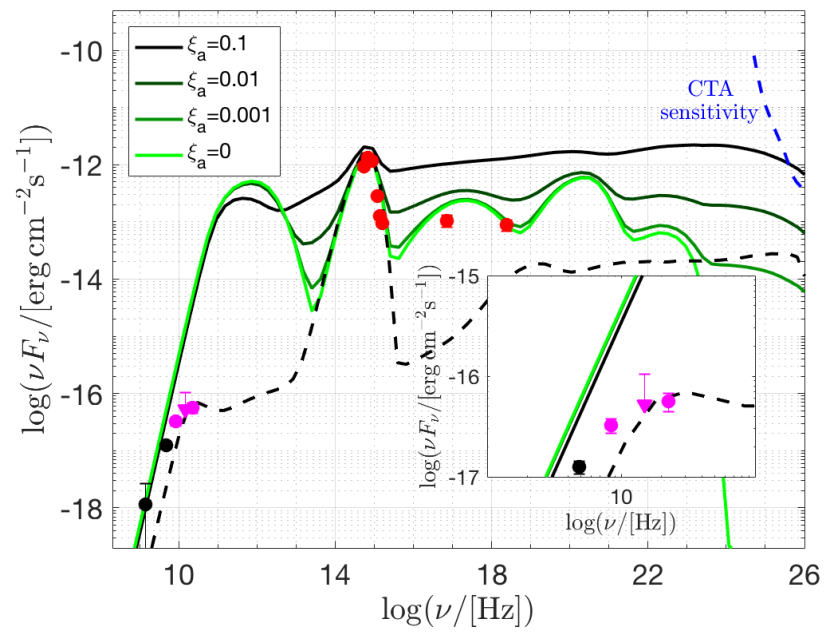

Figure 2: Simulated spectra for the forward shock at $\sim 3$ days compared to the data of GRB 060218 for a blast wave energy of $10^{51} \mathrm{erg}$ (solid lines) and $10^{49} \mathrm{erg}$ (dashed line). The spectra are made for varying number fraction of accelerated electrons $\xi_{\mathrm{a}}$. The inset shows a zoom-in on the radio band. Data points are taken from Campana et al. [3], Kaneko et al. [17], and Soderberg et al. [13], while the CTA sensitivity curve is taken from The CTA Collaboration [19].

Figures 2 and 3 show that the afterglow of GRB 060218 cannot have been as energetic as $10^{51} \mathrm{erg}$. Since the radio luminosities of other LLGRBs are comparable to that of GRB 060218, this results similarly applies to them. Thus, the afterglows of LLGRBs are not energetic enough be the main sources of UHECRs observed at Earth, even if UHECR acceleration does take place. This result is independent on whether the acceleration occurs on the reverse or the forward shock of the blast wave, as it is the total energy has been constrained. The analysis of this section constrains the mildly relativistic component of the outflow with $\Gamma \gtrsim 2$, that is decelerated in the first few days. If a transrelativistic component with velocity $\sim 0.3 c$ exists, where $c$ is the speed of light in vacuum, this material does not start to decelerate until weeks after the explosion and is therefore not constrained by our analysis. If the transrelavistic component carries a substantial energy ( $\left.\gtrsim 10^{51} \mathrm{erg}\right)$, this could still be a viable UHECR candidate.

\section{Conclusion}

We conclude that GRB 060218-like transients are unlikely to be the main sources of UHECRs. If acceleration occurs during the prompt phase, the optical luminosity would be orders of magnitude brighter than what was observed for GRB 060218. Acceleration could take place during the afterglow phase, but the afterglows are not energetic enough to supply the flux that we observe. Additionally, our analysis demonstrates that early radio observations can help constrain the kinetic energy in GRB afterglows and give us insight into the microphysics of mildly relativistic shocks. 


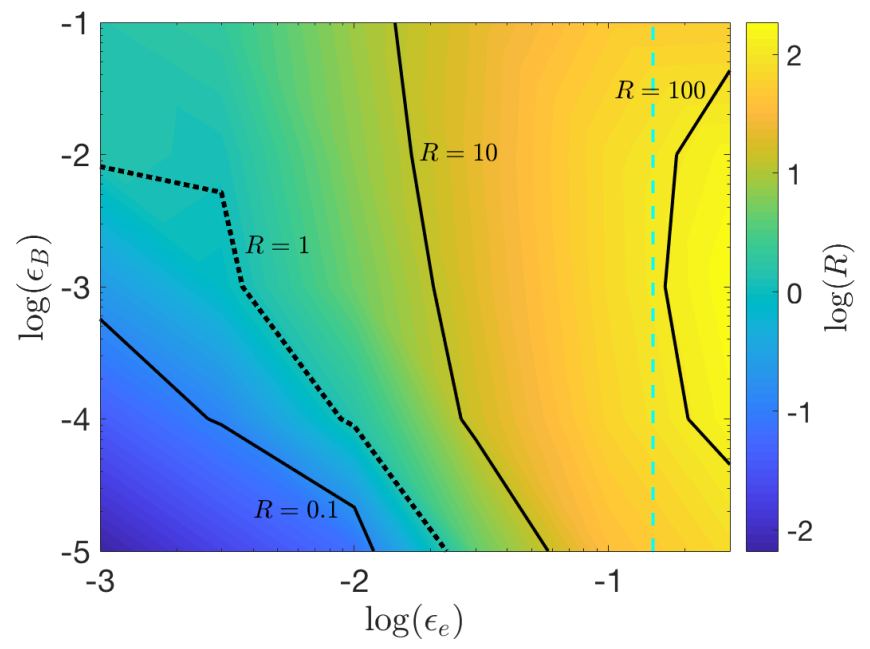

Figure 3: The ratio of the radio flux from the simulations compared to the data (see equation (1)) as a function of $\epsilon_{e}$ and $\epsilon_{B}$. The vertical cyan line shows the value of $\epsilon_{e}$ found by PIC simulations of mildly relativistic shocks [18]. With this value of $\epsilon_{e}$, the radio data, including its $1 \sigma$ error, would be overshone by almost two orders of magnitude.

\section{References}

[1] Samuelsson, F., Bégué, D., Ryde, F., \& Pe’er, A. 2019, ApJ, 876, 93

[2] Samuelsson, F., Bégué, D., Ryde, F., et al. 2020, ApJ, 902, 148

[3] Campana, S., Mangano, V., Blustin, A. J., et al. 2006, Natur, 442, 1008

[4] Sollerman, J., Jaunsen, A. O., Fynbo, J. P. U., et al. 2006, A\&A, 454, 503

[5] Murase, K., Ioka, K., Nagataki, S., \& Nakamura T. 2006, ApJL, 651, L5

[6] Starling, R. L. C, Wiersema, K., Levan, A. J., et al. 2011, MNRAS, 411, 2792

[7] Margutti, R., Soderberg, A. M., Wieringa, M. H., et al. 2013, ApJ, 778, 18

[8] D’Elia, V., Campana, S., D’Aì, A., et al. 2018, A\&A, 619, A66

[9] Sari, R., Piran, T., Narayan, R. 1998, ApJL, 497, L17

[10] Ghisellini, G., Ghirlanda, G., Tavecchio, F. 2007, MNRAS, 375, L36

[11] The Pierre Auger Collaboration. 2020, PhRvL, 125, 121106

[12] Fan, Y. Z., Piran, T., Xu, D. 2006, JCAP, 9, 013

[13] Soderberg, A. M., Kulkarni, S. R., Nakar, E., et al. 2006, Natur, 442, 1014

[14] Toma, K., Ioka, K., Sakamoto, T., Nakamura, T. 2007, Natur, 659, 1420 
[15] Eichler, D., Waxman, E. 2005, ApJ, 627, 861

[16] Pe'er, A., Waxman, E. 2005, ApJ, 628, 857

[17] Kaneko, Y., Ramirez-Ruiz, E., Granot, J., et al. 2007, ApJ, 654, 385

[18] Crumley, P., Caprioli, D., Markoff, S., Spitkovsky, A. 2019, MNRAS, 485, 5105

[19] Actis. M., Agnetta. G., Aharonian. F., et al. 2011, ExA, 32, 193 